\title{
Cooperative NOMA With Incremental Relaying: Performance Analysis and Optimization
}

Guoxin Li, Deepak Mishra and Hai J iang

The self-archived postprint version of this journal article is available at Linköping University Institutional Repository (DiVA):

http:/ / urn.kb.se/ resolve?urn=urn:nbn:se:liu:diva-153184

N.B.: When citing this work, cite the original publication.

Li, G., Mishra, D., Jiang, H., (2018), Cooperative NOMA With Incremental Relaying: Performance Analysis and Optimization, IEEE Transactions on Vehicular Technology, 67(11), 11291-11295. https:// doi.org/ 10.1109/TVT.2018.2869531

Original publication available at:

https:// doi.org/ 10.1109/TVT.2018.2869531

Copyright: Institute of Electrical and Electronics Engineers (IEEE)

http:// www.ieee.org/ index.html

(C) 2018 IEEE. Personal use of this material is permitted. However, permission to reprint/ republish this material for advertising or promotional purposes or for creating new collective works for resale or redistribution to servers or lists, or to reuse any copyrighted component of this work in other works must be obtained from the IEEE. 


\title{
Cooperative NOMA with Incremental Relaying: Performance Analysis and Optimization
}

\author{
Guoxin Li, Deepak Mishra, Member, IEEE, and Hai Jiang, Senior Member, IEEE
}

\begin{abstract}
In conventional cooperative non-orthogonal multiple access (NOMA) networks, spectral efficiency loss occurs due to a half-duplex constraint. To address this issue, we propose an incremental cooperative NOMA (ICN) protocol for a twouser downlink network. In particular, this protocol allows the source to adaptively switch between a direct NOMA transmission mode and a cooperative NOMA transmission mode according to a 1-bit feedback from the far user. We analytically prove that the proposed ICN protocol outperforms the conventional cooperative NOMA protocol. In addition, an optimal power allocation strategy at the source is studied to minimize the asymptotic system outage probability. Finally, numerical results validate our theoretical analysis, present insights, and quantify the enhancement achieved over the benchmark scheme.
\end{abstract}

Index Terms-Diversity order, incremental relaying, nonorthogonal multiple access, optimal power allocation, outage probability.

\section{INTRODUCTION}

Due to the ability to serve multiple users simultaneously in a single resource block, non-orthogonal multiple access (NOMA) is a viable solution to fulfill the fifth-generation (5G) wireless networks' requirements of high spectrum efficiency (SE) and massive connectivity [1]. Accordingly, NOMA has been included in the study item on $5 \mathrm{G}$ new radio (NR) by 3GPP in its Release 15 [2].

A typical scenario of NOMA is that, when a source needs to send signals to two users (e.g., in a downlink cellular system), it sends both signals simultaneously as a superimposed signal. The user with better channel condition (the strong user) first decodes the weak user's signal, and then performs successive interference cancellation (SIC) and decodes its own signal. The weak user decodes its own signal directly. Since the strong user decodes the weak user's signal first, the work in [3] proposes a cooperative NOMA protocol in which the strong user works as a half-duplex (HD) relay to help the weak user. This conventional cooperative NOMA $(\mathrm{CCN})$ protocol [3] promises to improve the weak user's performance by introducing a diversity gain. However, since the HD relay (the strong user) needs half of its time to forward information, the $\mathrm{CCN}$ protocol makes inefficient use of the degrees of freedom (DoF) of the channel and may cause a loss of SE (compared to a non-cooperative NOMA network). To efficiently exploit the DoF of the channel in a two-user downlink NOMA (TUDN) network, the work in [4] proposes a new cooperative protocol, termed as relaying with NOMA backhaul (R-NB). In this protocol, the source can adaptively adjust the time durations of NOMA transmission and relay transmission based

Manuscript received January 21, 2018; revised May 30, 2018; accepted August 29, 2018. The review of this paper was coordinated by Dr. K. Adachi.

G. Li and H. Jiang are with the Department of Electrical and Computer Engineering, University of Alberta, Edmonton, AB T6G 1H9, Canada (e-mail: guoxin@ualberta.ca, hai1@ualberta.ca).

D. Mishra is with the Division of Communication Systems, Department of Electrical Engineering (ISY), Linköping University, Linköping 58183, Sweden (e-mail: deepak.mishra@liu.se). on global instantaneous channel state information (CSI). However, global instantaneous CSI at the source may be difficult or costly to obtain in practice. This observation motivates us to propose a new and practically viable cooperative protocol for a TUDN network to improve SE of the CCN protocol.

Recall that in conventional cooperative networks, the incremental relaying (IR) protocol [5] is widely adopted since it can achieve higher SE by introducing a negligible 1-bitfeedback overhead. Specifically, the IR protocol invokes a relay for cooperation only when the source-to-destination channel gain is below a predetermined threshold. Inspired by this feature, in this correspondence we propose an incremental cooperative NOMA (ICN) protocol for a TUDN network with only statistical CSI at the source. In this protocol, the strong user works as a HD relay only when the weak user broadcasts a 1-bit negative feedback. The main contributions of this correspondence can be summarized as follows. 1) We propose a new and practical cooperative protocol for TUDN networks. To the best of our knowledge, the proposed ICN is the first time that the IR protocol is introduced into NOMA networks. 2) For the proposed ICN protocol, we derive exact or tightly approximated closed-form expressions of the outage probability (OP) of each user and the overall system. We prove that the ICN protocol outperforms the $\mathrm{CCN}$ protocol in terms of each user's OP and the system OP (SOP). 3) Asymptotic outage behavior of the ICN protocol is studied to derive the diversity order of each user and the optimal power allocation (OPA) strategy that minimizes the SOP. 4) Valuable insights regarding the ICN protocol are provided through detailed theoretical analysis and numerical results.

\section{System Model}

We consider a TUDN scenario with a source (S) and two users: user $1\left(\mathrm{U}_{1}\right)$ is the near user while user $2\left(\mathrm{U}_{2}\right)$ is the far user. Similar to [6], [7], the two users are ordered according to their distance to $\mathrm{S}$. Thus, $\mathrm{U}_{1}$ and $\mathrm{U}_{2}$ are treated as the strong user and the weak user, respectively. All the channels suffer Rayleigh fading. Let $h_{1}, h_{2}$ and $h_{3}$ denote the channel coefficients from $\mathrm{S}$ to $\mathrm{U}_{1}, \mathrm{~S}$ to $\mathrm{U}_{2}$, and $\mathrm{U}_{1}$ to $\mathrm{U}_{2}$, respectively, where $h_{i} \sim \mathcal{C N}\left(0, \Omega_{i}\right)(i=1,2,3)$. We assume that channel coefficients remain unchanged during one transmission block, but may vary from one transmission block to another. Next we introduce the proposed ICN protocol in details.

\section{A. Incremental Cooperative NOMA Protocol}

At the beginning of each transmission block, $\mathrm{S}$ broadcasts a pilot signal to $\mathrm{U}_{1}$ and $\mathrm{U}_{2}$. Based on the received pilot signal, $\mathrm{U}_{2}$ performs channel estimation of $h_{2}$ and compares it with a predefined threshold. If $\mathrm{U}_{2}$ judges that it can correctly decode its desired message through direct transmission, it feedbacks a 1-bit positive acknowledgement (ACK) to $S$ and $\mathrm{U}_{1}$. After receiving the ACK feedback, $\mathrm{S}$ adopts a direct NOMA transmission (DNT) mode, i.e., it sends the superimposed signal 


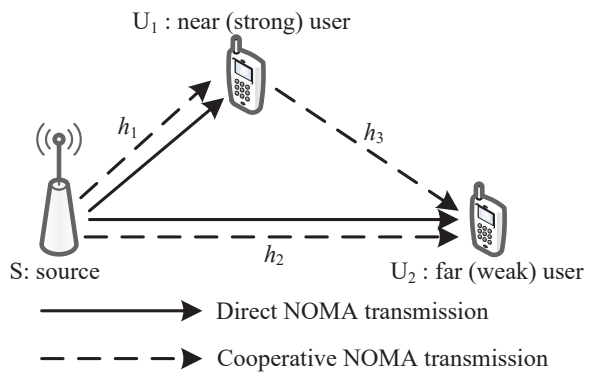

Fig. 1. System model.

to $\mathrm{U}_{1}$ and $\mathrm{U}_{2}$ within the whole transmission block. If $\mathrm{U}_{2}$ finds that it is unable to decode its desired message without $\mathrm{U}_{1}$ 's cooperation, it feedbacks a 1-bit negative acknowledge (NACK) to $\mathrm{S}$ and $\mathrm{U}_{1}$. Upon hearing the NACK feedback, $\mathrm{S}$ adopts a cooperative NOMA transmission (CNT) mode, i.e., it broadcasts the superimposed signal in the first half of the transmission block, and then $\mathrm{U}_{1}$ decodes $\mathrm{U}_{2}$ 's message and forwards it in the second half of the transmission block.

To identify the difference between our proposed ICN and the $\mathrm{CCN}$ protocols, here we briefly review the $\mathrm{CCN}$ protocol [3]. In the CCN protocol, the transmission block is divided into two phases with equal duration. During the first phase, $\mathrm{S}$ sends the superimposed signal to $\mathrm{U}_{1}$ and $\mathrm{U}_{2}$, and $\mathrm{U}_{1}$ decodes $\mathrm{U}_{2}$ 's message and forwards it in the second phase. Compared to the $\mathrm{CCN}$ protocol, our proposed ICN protocol is essentially an adaptive protocol which can adaptively switch between the DNT mode and the CNT mode based on a 1-bit indicator. ${ }^{1}$

\section{B. Signal Model}

1) DNT Mode: $\mathrm{S}$ sends a superimposed signal to $\mathrm{U}_{1}$ and $\mathrm{U}_{2}$, which occupies the whole transmission block. The resulted signal at $\mathrm{U}_{n}$ is defined by

$$
y_{n}=\sqrt{\alpha_{1} P_{s}} h_{n} x_{1}+\sqrt{\alpha_{2} P_{s}} h_{n} x_{2}+w_{n}, n=1,2,
$$

where $P_{s}$ is the transmit power of $\mathrm{S}, x_{n}$ denotes the message for $\mathrm{U}_{n}, \alpha_{n}$ is the power allocation (PA) factor for $x_{n}$ with $\alpha_{1}+\alpha_{2}=1$, and $w_{n}$ is the additive white Gaussian noise (AWGN) at $\mathrm{U}_{n}$ with zero mean and variance $\sigma^{2}$.

According to the NOMA principle, $\mathrm{U}_{n}$ first decodes $x_{2}$ upon observing $y_{n}$. Denote $\gamma_{n, 2}$ as the received signal-tointerference-pulse-noise ratio (SINR) at $\mathrm{U}_{n}$ to decode $x_{2}$, and then $\gamma_{n, 2}$ is given by $\gamma_{n, 2}=\frac{\alpha_{2} \rho_{s}\left|h_{n}\right|^{2}}{\alpha_{1} \rho_{s}\left|h_{n}\right|^{2}+1}$, where $\rho_{s}=P_{s} / \sigma^{2}$ denotes the transmit signal-to-noise ratio (SNR) of S. After $\mathrm{U}_{1}$ successfully decodes $x_{2}$ and performs SIC, the received SNR to detect $x_{1}$ at $\mathrm{U}_{1}$, denoted by $\gamma_{1,1}$, is $\gamma_{1,1}=\alpha_{1} \rho_{s}\left|h_{1}\right|^{2}$.

2) CNT Mode: Here the entire transmission block consists of two phases with equal duration. In the first phase, the received signal at $\mathrm{U}_{n}$ is the same as defined in (1), and the received SINR at $\mathrm{U}_{n}$ for message $x_{2}$ is also given as $\gamma_{n, 2}$ defined in the DNT mode. If $\mathrm{U}_{1}$ successfully decodes $x_{2}$ and performs

\footnotetext{
${ }^{1}$ In the $\mathrm{CCN}$ protocol, both $\mathrm{S}$ and $\mathrm{U}_{1}$ need to send pilot signals, for channel estimation at the receiver side(s). In the ICN protocol, only $\mathrm{S}$ sends a pilot signal in the DNT mode, while both $\mathrm{S}$ and $\mathrm{U}_{1}$ send pilot signals in the CNT mode. Thus, the signaling overhead of the two protocols are comparable to each other.
}

SIC in the first phase, its received SNR to detect $x_{1}$ is given as $\gamma_{1,1}$ defined in the DNT mode. Then, in the second phase, $\mathrm{U}_{1}$ forwards the re-encoded $x_{2}$ to $\mathrm{U}_{2}$. The corresponding received signal at $\mathrm{U}_{2}$ in the second phase can be expressed as $y_{2}^{\prime}=$ $\sqrt{P_{r}} h_{3} x_{2}+w_{2}$, where $P_{r}$ is the transmit power of $\mathrm{U}_{1}$. Finally, $\mathrm{U}_{2}$ combines the observed signals $y_{2}$ and $y_{2}^{\prime}$ using the maximal ratio combining (MRC), and thus, the received SINR at $\mathrm{U}_{2}$ to decode $x_{2}$ after MRC is given by $\gamma_{2,2}^{\mathrm{MRC}}=\frac{\alpha_{2} \rho_{s}\left|h_{2}\right|^{2}}{\alpha_{1} \rho_{s}\left|h_{2}\right|^{2}+1}+$ $\rho_{r}\left|h_{3}\right|^{2}$, where $\rho_{r}=P_{r} / \sigma^{2}$ is $\mathrm{U}_{1}$ 's transmit SNR.

\section{Outage Performance Analysis And OPTIMIZATION}

For each user, an outage event happens when the received SINR (or SNR) is below a pre-determined decoding threshold. Note that the decoding thresholds of the DNT and the CNT modes are different. In the DNT mode, the decoding threshold is $\gamma_{\text {th }}=2^{R}-1$ with $R$ being the target rate of $x_{1}$ and $x_{2}$. In the CNT mode, the threshold is $\gamma_{\text {th }}^{\prime}=2^{2 R}-1$.

\section{A. Outage Probability Analysis}

1) Near User: According to the ICN protocol, the OP of $\mathrm{U}_{1}$ can be expressed as

$$
\begin{aligned}
P_{1}^{\mathrm{ICN}}=1 & -\operatorname{Pr}\left\{\gamma_{2,2} \geq \gamma_{\mathrm{th}}, \gamma_{1,2} \geq \gamma_{\mathrm{th}}, \gamma_{1,1} \geq \gamma_{\mathrm{th}}\right\} \\
& -\operatorname{Pr}\left\{\gamma_{2,2}<\gamma_{\mathrm{th}}, \gamma_{1,2} \geq \gamma_{\mathrm{th}}^{\prime}, \gamma_{1,1} \geq \gamma_{\mathrm{th}}^{\prime}\right\},
\end{aligned}
$$

where $\operatorname{Pr}\{\cdot\}$ means probability of an event, $\gamma_{2,2} \geq \gamma_{\text {th }}$ indicates that the system works in the DNT mode, and $\gamma_{2,2}<\gamma_{\text {th }}$ indicates that the system works in the CNT mode. As $\gamma_{2,2}$ is independent from $\gamma_{1,2}$ and $\gamma_{1,1}$, (2) can be rewritten as

$$
\begin{aligned}
P_{1}^{\mathrm{ICN}}=1 & -\underbrace{\operatorname{Pr}\left\{\gamma_{2,2} \geq \gamma_{\mathrm{th}}\right\}}_{Q_{1}} \underbrace{\operatorname{Pr}\left\{\gamma_{1,2} \geq \gamma_{\mathrm{th}}, \gamma_{1,1} \geq \gamma_{\mathrm{th}}\right\}}_{\bar{Q}_{1}} \\
& -\underbrace{\operatorname{Pr}\left\{\gamma_{2,2}<\gamma_{\mathrm{th}}\right\}}_{Q_{2}} \underbrace{\operatorname{Pr}\left\{\gamma_{1,2} \geq \gamma_{\mathrm{th}}^{\prime}, \gamma_{1,1} \geq \gamma_{\mathrm{th}}^{\prime}\right\}}_{Q_{3}},
\end{aligned}
$$

where $\bar{Q}_{1}=1-Q_{1}$. It is easy to verify that $Q_{1}=Q_{2}=0$ for $\frac{1}{1+\gamma_{\text {th }}} \leq \alpha_{1}<1$, and $Q_{3}=0$ for $\frac{1}{1+\gamma_{\text {th }}^{\prime}} \leq \alpha_{1}<1$. Thus, $P_{1}^{\mathrm{ICN}}=1$ for $\frac{1}{1+\gamma_{\mathrm{th}}} \leq \alpha_{1}<1$. When $0<\alpha_{1}<\frac{1}{1+\gamma_{\mathrm{th}}}, Q_{2}$ is given by

$$
\begin{aligned}
Q_{2} & =\operatorname{Pr}\left\{\left|h_{1}\right|^{2} \geq \frac{\gamma_{\text {th }}}{\rho_{s}\left(\alpha_{2}-\gamma_{\text {th }} \alpha_{1}\right)},\left|h_{1}\right|^{2} \geq \frac{\gamma_{\text {th }}}{\alpha_{1} \rho_{s}}\right\} \\
& =\operatorname{Pr}\left\{\left|h_{1}\right|^{2} \geq \frac{\gamma_{\text {th }}}{\rho_{s} \Theta}\right\}=e^{-\frac{\gamma_{\text {th }}}{\rho_{s} \Omega_{1} \Theta}},
\end{aligned}
$$

where $\Theta \triangleq \min \left\{\theta, \alpha_{1}\right\}$ and $\theta \triangleq \alpha_{2}-\gamma_{\mathrm{th}} \alpha_{1} . Q_{2}$ is derived using the fact that $\left|h_{i}\right|^{2}(i=1,2,3)$ follows exponential distribution with mean $\Omega_{i}$. Following similar steps, we have $Q_{1}=e^{-\frac{\gamma_{\mathrm{th}}}{\rho_{s} \Omega_{2} \theta}}$ for $0<\alpha_{1}<\frac{1}{1+\gamma_{\mathrm{th}}}$, and $Q_{3}=e^{-\frac{\gamma_{\mathrm{th}}^{\prime}}{\rho_{s} \Omega_{1} \Theta^{\prime}}}$ for $0<\alpha_{1}<\frac{1}{1+\gamma_{\text {th }}^{\prime}}$, where $\Theta^{\prime} \triangleq \min \left\{\theta^{\prime}, \alpha_{1}\right\}$ and $\theta^{\prime} \triangleq \alpha_{2}-\gamma_{\mathrm{th}}^{\prime} \alpha_{1}$. Substituting the results of $Q_{1}, Q_{2}$ and $Q_{3}$ into (3), a closed-form expression of $\mathrm{U}_{1}$ 's $\mathrm{OP}$ is given by

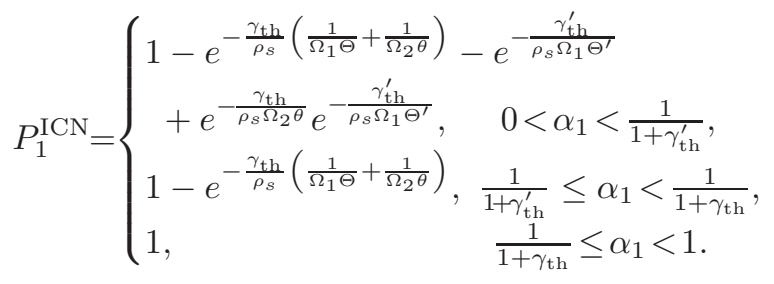


2) Far User: The OP of $\mathrm{U}_{2}$ with the ICN protocol is given by

$$
\begin{aligned}
P_{2}^{\mathrm{ICN}}= & \operatorname{Pr}\left\{\gamma_{2,2}<\gamma_{\mathrm{th}}, \gamma_{1,2}<\gamma_{\mathrm{th}}^{\prime}\right\} \\
& +\operatorname{Pr}\left\{\gamma_{2,2}<\gamma_{\mathrm{th}}, \gamma_{1,2} \geq \gamma_{\mathrm{th}}^{\prime}, \gamma_{2,2}^{\mathrm{MRC}}<\gamma_{\mathrm{th}}^{\prime}\right\} \\
= & \underbrace{\operatorname{Pr}\left\{\gamma_{2,2}<\gamma_{\mathrm{th}}\right\}}_{\bar{Q}_{1}} \underbrace{\operatorname{Pr}\left\{\gamma_{1,2}<\gamma_{\mathrm{th}}^{\prime}\right\}}_{Q_{4}} \\
& +\underbrace{\operatorname{Pr}\left\{\gamma_{1,2} \geq \gamma_{\mathrm{th}}^{\prime}\right\}}_{\bar{Q}_{4}} \underbrace{\operatorname{Pr}\left\{\gamma_{2,2}<\gamma_{\mathrm{th}}, \gamma_{2,2}^{\mathrm{MRC}}<\gamma_{\mathrm{th}}^{\prime}\right\}}_{Q_{5}},
\end{aligned}
$$

where $\bar{Q}_{4}=1-Q_{4}$. Similar to $\mathrm{U}_{1}$ 's $\mathrm{OP}$, the OP of $\mathrm{U}_{2}$ is also segmented regarding $\alpha_{1}$ as follows.

When $\frac{1}{1+\gamma_{\mathrm{th}}} \leq \alpha_{1}<1$, we have $P_{2}^{\mathrm{ICN}}=1$ since $\bar{Q}_{1}=$ $Q_{4}=1$.

When $\frac{1}{1+\gamma_{\text {th }}^{\prime}} \leq \alpha_{1}<\frac{1}{1+\gamma_{\text {th }}}$, we have $Q_{4}=1$ and thus, $P_{2}^{\mathrm{ICN}}=\bar{Q}_{1}=1-e^{-\frac{\gamma_{\mathrm{th}}}{\rho_{s} \Omega_{2} \theta}}$, which is an increasing function of $\alpha_{1}$.

Now we derive $P_{2}^{\mathrm{ICN}}$ over the region $\alpha_{1} \in\left(0, \frac{1}{1+\gamma_{\mathrm{th}}^{\prime}}\right)$, where $Q_{4}=1-e^{-\frac{\gamma_{\text {th }}^{\prime}}{\rho_{s} \Omega_{1} \theta^{\prime}}}$ and $Q_{5}$ can be derived as

$$
\begin{aligned}
Q_{5} & =\operatorname{Pr}\left\{\frac{\alpha_{2} \rho_{s}\left|h_{2}\right|^{2}}{\alpha_{1} \rho_{s}\left|h_{2}\right|^{2}+1}<\gamma_{\mathrm{th}}, \rho_{r}\left|h_{3}\right|^{2}+\frac{\alpha_{2} \rho_{s}\left|h_{2}\right|^{2}}{\alpha_{1} \rho_{s}\left|h_{2}\right|^{2}+1}<\gamma_{\mathrm{th}}^{\prime}\right\} \\
& =\int_{0}^{\frac{\gamma_{\mathrm{th}}}{\rho_{s} \theta}} F_{\left|h_{3}\right|^{2}}\left(\frac{\gamma_{\mathrm{th}}^{\prime}}{\rho_{r}}-\frac{\alpha_{2} \rho_{s} x}{\rho_{r}\left(\alpha_{1} \rho_{s} x+1\right)}\right) f_{\left|h_{2}\right|^{2}}(x) d x \\
& =1-e^{-\frac{\gamma_{\mathrm{th}}}{\rho_{s} \Omega_{2} \theta}}-\underbrace{\int_{0}^{\frac{\gamma_{\mathrm{th}}}{\rho_{s} \theta}} e^{-\frac{1}{\rho_{r} \Omega_{3}}\left(\gamma_{\mathrm{th}}^{\prime}-\frac{\alpha_{2} \rho_{s} x}{\alpha_{1} \rho_{s} x+1}\right)} \frac{1}{\Omega_{2}} e^{-\frac{x}{\Omega_{2}}} d x}_{Q_{6}} .
\end{aligned}
$$

Though it is difficult to derive a closed-form expression for $Q_{6}$, we can obtain an approximation for it. By replacing the variable $x=\frac{\gamma_{\mathrm{th}}}{2 \rho_{s} \theta}(t+1)$ in $Q_{6}$ and using GaussianChebyshev quadrature [8, Eq. 25.4.38], we have

$$
\begin{aligned}
Q_{6} & =\frac{\gamma_{\mathrm{th}}}{2 \rho_{s} \Omega_{2} \theta} \int_{-1}^{1} e^{-\frac{g(t)}{\rho_{r} \Omega_{3}}} e^{-\frac{\gamma_{\mathrm{th}}(t+1)}{2 \rho_{s} \Omega_{2} \theta}} d t \\
& \approx \frac{\gamma_{\mathrm{th}}}{2 \rho_{s} \Omega_{2} \theta} \frac{\pi}{K} \sum_{k=1}^{K} \sqrt{1-\xi_{k}^{2}} e^{-\frac{g\left(\xi_{k}\right)}{\rho_{r} \Omega_{3}}} e^{-\frac{\gamma_{\mathrm{th}}\left(\xi_{k}+1\right)}{2 \rho_{s} \Omega_{2} \theta}}
\end{aligned}
$$

where $K$ is a parameter to balance accuracy and complexity, $\xi_{k}=\cos \left(\frac{2 k-1}{2 K} \pi\right)$, and $g(x)=\gamma_{\text {th }}^{\prime}-\frac{\gamma_{\mathrm{th}}(x+1) \alpha_{2}}{\gamma_{\mathrm{th}}(x+1) \alpha_{1}+2 \theta}$. Substituting (8) into (7), we can obtain an approximation of $Q_{5}$.

Combining the results for $Q_{1}, Q_{4}$ and $Q_{5}$, and after some algebraic manipulations, a closed-form expression of approximated $P_{2}^{\mathrm{ICN}}$ over the region $\alpha_{1} \in\left(0, \frac{1}{1+\gamma_{\text {th }}^{\prime}}\right)$ is given by

$$
P_{2}^{\mathrm{ICN}} \approx 1-e^{-\frac{\gamma_{\text {th }}}{\rho_{s} \Omega_{2} \theta}}-e^{-\frac{\gamma_{\text {th }}^{\prime}}{\rho_{s} \Omega_{1} \theta^{\prime}}} Q_{6},
$$

where $Q_{6}$ is given by (8).

From the above derivations, we know that $P_{1}^{\mathrm{ICN}}$ and $P_{2}^{\mathrm{ICN}}$ are both equal to 1 when $\frac{1}{1+\gamma_{\text {th }}} \leq \alpha_{1}<1$. Thus, in the sequel we only focus on the remaining region, i.e., $0<\alpha_{1}<\frac{1}{1+\gamma_{\text {th }}}$.
3) Overall System: Similar to [3], the system outage is defined as the event when one user or both users in the system are in outage. Thus, the SOP with the ICN protocol can be expressed as

$$
\begin{aligned}
P_{1 \& 2}^{\mathrm{ICN}} & =1-\operatorname{Pr}\left\{\gamma_{2,2} \geq \gamma_{\mathrm{th}}, \gamma_{1,2} \geq \gamma_{\mathrm{th}}, \gamma_{1,1} \geq \gamma_{\mathrm{th}}\right\} \\
& -\operatorname{Pr}\left\{\gamma_{2,2}<\gamma_{\mathrm{th}}, \gamma_{1,2} \geq \gamma_{\mathrm{th}}^{\prime}, \gamma_{1,1} \geq \gamma_{\mathrm{th}}^{\prime}, \gamma_{2,2}^{\mathrm{MRC}} \geq \gamma_{\mathrm{th}}^{\prime}\right\}
\end{aligned}
$$

Following similar procedures to those in the derivations of $P_{1}^{\mathrm{ICN}}$ and $P_{2}^{\mathrm{ICN}}$, a closed-form approximation of the SOP can be given as

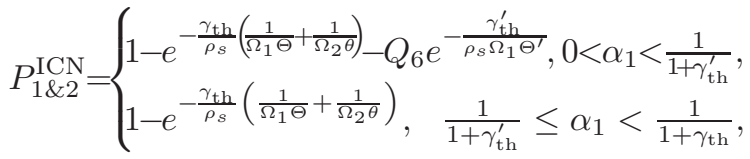

where $Q_{6}$ is given by (8). Comparing the expressions of $P_{1}^{\mathrm{ICN}}$ and $P_{1 \& 2}^{\mathrm{ICN}}$ given in (5) and (11), respectively, we find that the OP of $\mathrm{U}_{1}$ is identical to the SOP when $\alpha_{1} \in\left[\frac{1}{1+\gamma_{\text {th }}^{\prime}}, \frac{1}{1+\gamma_{\text {th }}}\right)$. In other words, when the overall system is in outage, it also means that $U_{1}$ is in outage. This is due to the following two facts: 1) The system works in the DNT mode only when $\mathrm{U}_{2}$ can correctly decode its desired information (which means that $\mathrm{U}_{2}$ has no outage). In this case, $\mathrm{U}_{1}$ in outage also leads to an outage of the overall system. 2) When $\mathrm{U}_{2}$ requests cooperation (which indicates that the target rate of $\mathrm{U}_{2}$ cannot be achieved in the DNT mode), if $\alpha_{1} \in\left[\frac{1}{1+\gamma_{\text {th }}^{\prime}}, \frac{1}{1+\gamma_{\text {th }}}\right)$, we have $\gamma_{1,2}<$ $\gamma_{\text {th }}^{\prime}$, i.e., $\mathrm{U}_{1}$ fails to decode $x_{2}$, which results in an outage at both $\mathrm{U}_{1}$ and $\mathrm{U}_{2}$.

\section{B. Outage Performance Comparison with the CCN protocol}

We denote the OP of $\mathrm{U}_{1}, \mathrm{U}_{2}$, and the overall system in the $\mathrm{CCN}$ protocol by $P_{1}^{\mathrm{CCN}}, P_{2}^{\mathrm{CCN}}$ and $P_{1 \& 2}^{\mathrm{CCN}}$, respectively. Following the $\mathrm{CCN}$ protocol details from [3] along with the expressions of $P_{1}^{\mathrm{ICN}}, P_{2}^{\mathrm{ICN}}$ and $P_{1 \& 2}^{\mathrm{ICN}}$ given in (2), (6) and (10), respectively, we have

$$
\begin{aligned}
P_{1}^{\mathrm{ICN}}<1 & -\operatorname{Pr}\left\{\gamma_{2,2} \geq \gamma_{\mathrm{th}}, \gamma_{1,2} \geq \gamma_{\mathrm{th}}^{\prime}, \gamma_{1,1} \geq \gamma_{\mathrm{th}}^{\prime}\right\} \\
& -\operatorname{Pr}\left\{\gamma_{2,2}<\gamma_{\mathrm{th}}, \gamma_{1,2} \geq \gamma_{\mathrm{th}}^{\prime}, \gamma_{1,1} \geq \gamma_{\mathrm{th}}^{\prime}\right\} \\
= & 1-\operatorname{Pr}\left\{\gamma_{1,2} \geq \gamma_{\mathrm{th}}^{\prime}, \gamma_{1,1} \geq \gamma_{\mathrm{th}}^{\prime}\right\}=P_{1}^{\mathrm{CCN}}, \\
P_{2}^{\mathrm{ICN}}< & \operatorname{Pr}\left\{\gamma_{2,2}<\gamma_{\mathrm{th}}^{\prime}, \gamma_{1,2}<\gamma_{\mathrm{th}}^{\prime}\right\} \\
& +\operatorname{Pr}\left\{\gamma_{1,2} \geq \gamma_{\mathrm{th}}^{\prime}, \gamma_{2,2}^{\mathrm{MRC}}<\gamma_{\mathrm{th}}^{\prime}\right\}=P_{2}^{\mathrm{CCN}},
\end{aligned}
$$

and

$$
\begin{aligned}
P_{1 \& 2}^{\mathrm{ICN}} & <1-\operatorname{Pr}\left\{\gamma_{2,2} \geq \gamma_{\mathrm{th}}, \gamma_{1,2} \geq \gamma_{\mathrm{th}}^{\prime}, \gamma_{1,1} \geq \gamma_{\mathrm{th}}^{\prime}, \gamma_{2,2}^{\mathrm{MRC}} \geq \gamma_{\mathrm{th}}^{\prime}\right\} \\
& -\operatorname{Pr}\left\{\gamma_{2,2}<\gamma_{\mathrm{th}}, \gamma_{1,2} \geq \gamma_{\mathrm{th}}^{\prime}, \gamma_{1,1} \geq \gamma_{\mathrm{th}}^{\prime}, \gamma_{2,2}^{\mathrm{MRC}} \geq \gamma_{\mathrm{th}}^{\prime}\right\} \quad(14) \\
& =1-\operatorname{Pr}\left\{\gamma_{1,2} \geq \gamma_{\mathrm{th}}^{\prime}, \gamma_{1,1} \geq \gamma_{\mathrm{th}}^{\prime}, \gamma_{2,2}^{\mathrm{MRC}} \geq \gamma_{\mathrm{th}}^{\prime}\right\}=P_{1 \& 2}^{\mathrm{CCN}} .
\end{aligned}
$$

Therefore, it can be concluded that the ICN protocol outperforms the CCN protocol in terms of each user's OP and the SOP.

\section{SOP Minimization and Diversity Order Analysis}

In this subsection, we first investigate the asymptotic outage performance of the ICN protocol when $\rho_{s} \rightarrow \infty$ and $\rho_{r}=\lambda \rho_{s}$ with $0<\lambda \leq 1$. Based on the asymptotic analysis, an OPA strategy that minimizes the SOP is developed, and the diversity order of each user is derived as well. 
1) SOP minimization: As $\rho_{s} \rightarrow \infty$, we have $\gamma_{2,2}^{\mathrm{MRC}} \rightarrow$ $\frac{\alpha_{2}}{\alpha_{1}}+\rho_{r}\left|h_{3}\right|^{2}>\gamma_{\text {th }}^{\prime}$ for $0<\alpha_{1}<\frac{1}{1+\gamma_{\text {th }}^{\prime}}$, which indicates that $\operatorname{Pr}\left\{\gamma_{2,2}^{\mathrm{MRC}}>\gamma_{\text {th }}^{\prime}\right\} \rightarrow 1$, and thus, $P_{1 \& 2}^{\mathrm{I} d \mathrm{~N}}$ converges to $P_{1}^{\mathrm{ICN}}$ based on (2) and (10). Together with the fact that $\mathrm{U}_{1}$ 's OP is identical to the SOP when $\alpha_{1} \in\left[\frac{1}{1+\gamma_{\text {th }}^{\prime}}, \frac{1}{1+\gamma_{\text {th }}}\right)$, it can be concluded that the SOP converges to $\mathrm{U}_{1}$ 's OP as $\rho_{s} \rightarrow \infty$. Noting this key observation, in the following we focus on the minimization of $\mathrm{U}_{1}$ 's OP.

When $\rho_{s} \rightarrow \infty$, applying $e^{-x} \stackrel{x \rightarrow 0}{\simeq} 1-x$ into (5), we can derive the asymptotic OP of $\mathrm{U}_{1}$ as

$$
P_{1, \text { asy }}^{\mathrm{ICN}} \simeq\left\{\begin{array}{l}
\frac{\gamma_{\mathrm{th}}}{\rho_{s} \Omega_{1} \Theta}+\frac{\gamma_{\mathrm{th}} \gamma_{\mathrm{h}}^{\prime}}{\rho_{s}^{2} \Omega_{1} \Omega_{2} \theta \Theta^{\prime}}, 0<\alpha_{1}<\frac{1}{1+\gamma_{\mathrm{th}}^{\prime}}, \\
\frac{\gamma_{\mathrm{th}}}{\rho_{s}}\left(\frac{1}{\Omega_{1} \Theta}+\frac{1}{\Omega_{2} \theta}\right), \frac{1}{1+\gamma_{\mathrm{th}}^{\prime}} \leq \alpha_{1}<\frac{1}{1+\gamma_{\mathrm{th}}} .
\end{array}\right.
$$

Substituting the expressions of $\theta, \Theta$, and $\Theta^{\prime}$ into (15), $P_{1, \text { asy }}^{\mathrm{ICN}}$ can be further expressed as

$$
P_{1, \mathrm{asy}}^{\mathrm{ICN}} \simeq\left\{\begin{array}{l}
\frac{\gamma_{\mathrm{th}}}{\rho_{s} \Omega_{1}} f_{1}\left(\alpha_{1}\right), 0<\alpha_{1}<\frac{1}{2+\gamma_{\text {th }}^{\prime}}, \\
\frac{\gamma_{\mathrm{th}}}{\rho_{s} \Omega_{1}} f_{2}\left(\alpha_{1}\right), \frac{1}{2+\gamma_{\text {th }}^{\prime}} \leq \alpha_{1}<\min \left\{\frac{1}{2+\gamma_{\mathrm{th}}}, \frac{1}{1+\gamma_{\text {th }}^{\prime}}\right\}, \\
\frac{\gamma_{\mathrm{th}}}{\rho_{s} \Omega_{1}} f_{3}\left(\alpha_{1}\right), \min \left\{\frac{1}{2+\gamma_{\mathrm{th}}}, \frac{1}{1+\gamma_{\text {th }}^{\prime}}\right\} \leq \alpha_{1}<\frac{1}{1+\gamma_{\text {th }}^{\prime}}, \\
\frac{\gamma_{\text {th }}}{\rho_{s}} f_{4}\left(\alpha_{1}\right), \frac{1}{1+\gamma_{\text {th }}^{\prime}} \leq \alpha_{1}<\max \left\{\frac{1}{2+\gamma_{\mathrm{th}}}, \frac{1}{1+\gamma_{\text {th }}^{\prime}}\right\}, \\
\frac{\gamma_{\text {th }}}{\rho_{s}} f_{5}\left(\alpha_{1}\right), \max \left\{\frac{1}{2+\gamma_{\mathrm{th}}}, \frac{1}{1+\gamma_{\mathrm{th}}^{\prime}}\right\} \leq \alpha_{1}<\frac{1}{1+\gamma_{\mathrm{th}}},
\end{array}\right.
$$

in which we have

$$
\begin{aligned}
& f_{1}\left(\alpha_{1}\right)=\frac{1}{\alpha_{1}}+\frac{\gamma_{\mathrm{th}}^{\prime}}{\rho_{s} \Omega_{2}\left(1-\alpha_{1}\left(1+\gamma_{\mathrm{th}}\right)\right) \alpha_{1}} \\
& f_{2}\left(\alpha_{1}\right)=\frac{1}{\alpha_{1}}+\frac{\gamma_{\mathrm{th}}^{\prime}}{\rho_{s} \Omega_{2}\left(1-\alpha_{1}\left(1+\gamma_{\mathrm{th}}\right)\right)\left(1-\alpha_{1}\left(1+\gamma_{\mathrm{th}}^{\prime}\right)\right)} \\
& f_{3}\left(\alpha_{1}\right)=\frac{1}{1-\alpha_{1}\left(1+\gamma_{\mathrm{th}}\right)}\left[1+\frac{\gamma_{\mathrm{th}}^{\prime}}{\rho_{s} \Omega_{2}\left(1-\alpha_{1}\left(1+\gamma_{\mathrm{th}}^{\prime}\right)\right)}\right] \\
& f_{4}\left(\alpha_{1}\right)=\frac{1}{\Omega_{1} \alpha_{1}}+\frac{1}{\Omega_{2}\left(1-\alpha_{1}\left(1+\gamma_{\mathrm{th}}\right)\right)} \\
& f_{5}\left(\alpha_{1}\right)=\frac{1}{1-\alpha_{1}\left(1+\gamma_{\mathrm{th}}\right)}\left(\frac{1}{\Omega_{1}}+\frac{1}{\Omega_{2}}\right)
\end{aligned}
$$

For $f_{1}\left(\alpha_{1}\right)$ : It can be shown that $\frac{1}{\alpha_{1}\left(1-\alpha_{1}\left(1+\gamma_{\mathrm{th}}\right)\right)}$ monotonically decreases with $\alpha_{1} \in\left(0, \frac{1}{2+2 \gamma_{\mathrm{th}}}\right)$. Thus, $f_{1}\left(\alpha_{1}\right)$ is a decreasing function over $\alpha_{1} \in\left(0, \frac{1}{2+\gamma_{\mathrm{th}}^{\prime}}\right)$ since $\frac{1}{2+\gamma_{\mathrm{th}}^{\prime}} \leq \frac{1}{2+2 \gamma_{\mathrm{th}}}$.

For $f_{2}\left(\alpha_{1}\right): f_{2}\left(\alpha_{1}\right)$ is a convex function of $\alpha_{1}$ due to the facts that $\frac{1}{\alpha_{1}}, \frac{1}{1-\alpha_{1}\left(1+\gamma_{\mathrm{th}}\right)}$ and $\frac{1}{1-\alpha_{1}\left(1+\gamma_{\mathrm{th}}^{\prime}\right)}$ are convex functions of $\alpha_{1}$ and that the sum of convex functions is still a convex function. The first-order derivative of $f_{2}\left(\alpha_{1}\right)$ is given by

$$
\frac{\mathrm{d} f_{2}\left(\alpha_{1}\right)}{\mathrm{d} \alpha_{1}}=-\frac{1}{\alpha_{1}^{2}}+\frac{a\left(b\left(1-c \alpha_{1}\right)+c\left(1-b \alpha_{1}\right)\right)}{\left(\left(1-b \alpha_{1}\right)\left(1-c \alpha_{1}\right)\right)^{2}},
$$

where $a=\frac{\gamma_{\mathrm{th}}^{\prime}}{\rho_{s} \Omega_{2}}, b=1+\gamma_{\mathrm{th}}$ and $c=1+\gamma_{\mathrm{th}}^{\prime}$. From (22), we can easily verify that $\left.\frac{\mathrm{d} f_{2}\left(\alpha_{1}\right)}{\mathrm{d} \alpha_{1}}\right|_{\alpha_{1} \rightarrow 0}<0$ and $\left.\frac{\mathrm{d} f_{2}\left(\alpha_{1}\right)}{\mathrm{d} \alpha_{1}}\right|_{\alpha_{1} \rightarrow \frac{1}{1+\gamma_{\text {th }}^{\prime}}}>0$. Since $f_{2}\left(\alpha_{1}\right)$ is a convex function, the critical point of $f_{2}\left(\alpha_{1}\right)$, denoted as $\delta$, must lie in the interval $\left(0, \frac{1}{1+\gamma_{\text {th }}^{\prime}}\right)$, and is the root of $\frac{\mathrm{d} f_{2}\left(\alpha_{1}\right)}{\mathrm{d} \alpha_{1}}=0$ that falls in $\left(0, \frac{1}{1+\gamma_{\text {th }}^{\prime}}\right) \cdot{ }^{2}$ Thus, for $\frac{1}{2+\gamma_{\text {th }}^{\prime}} \leq \alpha_{1}<\min \left\{\frac{1}{2+\gamma_{\text {th }}}, \frac{1}{1+\gamma_{\text {th }}^{\prime}}\right\}$, the minimal point of $P_{1, \text { asy }}^{\mathrm{ICN}}$ is at $\alpha_{1}=\beta_{1}$ with $\beta_{1} \triangleq$ $\max \left\{\frac{1}{2+\gamma_{\text {th }}^{\prime}}, \min \left\{\delta, \frac{1}{2+\gamma_{\mathrm{th}}}\right\}\right\}$.

For $f_{3}\left(\alpha_{1}\right): f_{3}\left(\alpha_{1}\right)$ is an increasing function of $\alpha_{1}$.

For $f_{4}\left(\alpha_{1}\right)$ : Like $f_{2}\left(\alpha_{1}\right), f_{4}\left(\alpha_{1}\right)$ is also a convex function of $\alpha_{1}$, whose critical point can be obtained as $\alpha_{1}=$ $\frac{1}{1+\psi+\gamma_{\mathrm{th}}}$, where $\psi=\sqrt{\frac{\Omega_{1}\left(1+\gamma_{\mathrm{th}}\right)}{\Omega_{2}}}$. Thus, for $\frac{1}{1+\gamma_{\mathrm{th}}^{\prime}} \leq \alpha_{1}<$ $\max \left\{\frac{1}{2+\gamma_{\mathrm{th}}}, \frac{1}{1+\gamma_{\mathrm{th}}^{\prime}}\right\}$, the minimal point of $P_{1, \text { asy }}^{\mathrm{ICN}}$ is at $\alpha_{1}=\beta_{2}$ with $\beta_{2} \triangleq \max \left\{\frac{1}{1+\psi+\gamma_{\text {th }}}, \frac{1}{1+\gamma_{\text {th }}^{\prime}}\right\}$.

For $f_{5}\left(\alpha_{1}\right): f_{5}\left(\alpha_{1}\right)$ is an increasing function of $\alpha_{1}$.

Combing all above observations, we conclude that $P_{1, \text { asy }}^{\mathrm{ICN}}$ achieves its global minimum value at $\alpha_{1}=\beta_{1}$ if $\frac{\gamma_{\text {th }}}{\rho_{s} \Omega_{1}} f_{2}\left(\beta_{1}\right)<$ $\frac{\gamma_{\text {th }}}{\rho_{s}} f_{4}\left(\beta_{2}\right)$, or at $\alpha_{1}=\beta_{2}$ otherwise.

2) Diversity order of each user: From (15), we can observe that the diversity order of $U_{1}$ is 1 , which is the full diversity order for $\mathrm{U}_{1}$.

As $\rho_{s} \rightarrow \infty$, the asymptotic OP of $\mathrm{U}_{2}$ over the region $\alpha_{1} \in\left[\frac{1}{1+\gamma_{\text {th }}^{\prime}}, \frac{1}{1+\gamma_{\text {th }}}\right)$ can be easily derived as $P_{2, \text { asy }}^{\mathrm{ICN}}=\bar{Q}_{1} \simeq$ $\frac{\gamma_{\text {th }}}{\rho_{s} \Omega_{2} \theta}$, which illustrates that the diversity order of $U_{2}$ in this region is 1 . The reason for the diversity loss is that in this region of $\alpha_{1}, \mathrm{U}_{1}$ cannot work in the cooperative mode since $\gamma_{1,2}<\gamma_{\text {th }}^{\prime}$, and thus, it fails to provide assistance to $\mathrm{U}_{2}$.

Now we focus on the derivation of $P_{2, \text { asy }}^{\mathrm{ICN}}$ when $0<\alpha_{1}<$ $\frac{1}{1+\gamma_{\mathrm{th}}^{\prime}}$. As $\rho_{s} \rightarrow \infty, Q_{6}$ in (7) can be approximated as

$$
\begin{aligned}
Q_{6} & \stackrel{(\mathrm{i})}{\simeq} \int_{0}^{\frac{\gamma_{\mathrm{th}}}{\rho_{s} \theta}}\left(1-\frac{1}{\rho_{r} \Omega_{3}}\left(\gamma_{\mathrm{th}}^{\prime}-\frac{\alpha_{2} \rho_{s} x}{\left(\alpha_{1} \rho_{s} x+1\right)}\right)\right) \frac{1}{\Omega_{2}} e^{-\frac{x}{\Omega_{2}}} d x \\
& \stackrel{(\mathrm{ii})}{\simeq}\left(1-e^{-\frac{\gamma_{\mathrm{th}}}{\rho_{s} \Omega_{2} \theta}}\right)\left(1-\frac{\gamma_{\mathrm{th}}^{\prime}}{\rho_{r} \Omega_{3}}\right)+\frac{\gamma_{\mathrm{th}}}{2 \rho_{s} \rho_{r} \Omega_{2} \Omega_{3} \theta} \frac{\pi}{K} \\
& \times \sum_{k=1}^{K} \sqrt{1-\xi_{k}^{2}} \frac{\alpha_{2} \gamma_{\mathrm{th}}\left(\xi_{k}+1\right)}{\alpha_{1} \gamma_{\mathrm{th}}\left(\xi_{k}+1\right)+2 \theta} e^{-\frac{\gamma_{\mathrm{th}}\left(\xi_{k}+1\right)}{2 \rho_{s} \Omega_{2} \theta}},
\end{aligned}
$$

where step (i) is obtained by using $e^{-x} \stackrel{x \rightarrow 0}{\simeq} 1-x$, and step (ii) is achieved by applying the Gaussian-Chebyshev quadrature. Now substituting (23) into (7) and applying $e^{-x} \stackrel{x \rightarrow 0}{\simeq} 1-x$ again, we have $Q_{5} \simeq \frac{\gamma_{\text {th }} \Xi}{\lambda \rho_{s}^{2} \Omega_{2} \Omega_{3} \theta}$, where $\Xi$ is given by

$$
\Xi=\gamma_{\text {th }}^{\prime}-\frac{\pi}{2 K} \sum_{k=1}^{K} \sqrt{1-\xi_{k}^{2}} \frac{\alpha_{2} \gamma_{\text {th }}\left(\xi_{k}+1\right)}{\alpha_{1} \gamma_{\text {th }}\left(\xi_{k}+1\right)+2 \theta} .
$$

In addition, an approximation of $\bar{Q}_{1} Q_{4}$ in (6) can be easily obtained as $\bar{Q}_{1} Q_{4} \simeq \frac{\gamma_{\text {th }} \gamma_{\text {th }}^{\prime}}{\rho_{s}^{2} \Omega_{1} \Omega_{2} \theta \theta^{\prime}}$. To this end, by combining the approximate results for $\bar{Q}_{1} Q_{4}$ and $Q_{5}$, the asymptotic OP of $\mathrm{U}_{2}$ over the region $\alpha_{1} \in\left(0, \frac{1}{1+\gamma_{\text {th }}^{\prime}}\right)$ is given by

$$
P_{2, \mathrm{asy}}^{\mathrm{ICN}} \simeq \frac{1}{\rho_{s}^{2}}\left(\frac{\gamma_{\mathrm{th}} \gamma_{\mathrm{th}}^{\prime}}{\Omega_{1} \Omega_{2} \theta \theta^{\prime}}+\frac{\gamma_{\mathrm{th}} \Xi}{\lambda \Omega_{2} \Omega_{3} \theta}\right)
$$

According to (25), it is clear that in region $\alpha_{1} \in\left(0, \frac{1}{1+\gamma_{\text {th }}^{\prime}}\right)$, $\mathrm{U}_{2}$ achieves its full diversity order of two.

${ }^{2}$ Note that $\frac{\mathrm{d} f_{2}\left(\alpha_{1}\right)}{\mathrm{d} \alpha_{1}}$ can be transformed to a quartic function of $\alpha_{1}$, and
the procedures in [9] can be used to find closed-form roots of $\frac{\mathrm{d} f_{2}\left(\alpha_{1}\right)}{\mathrm{d} \alpha_{1}}=0$. 


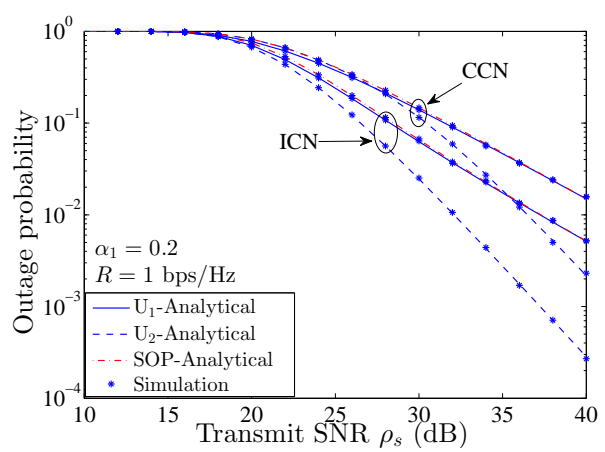

Fig. 2. Outage performance of the ICN and $\mathrm{CCN}$ protocols.

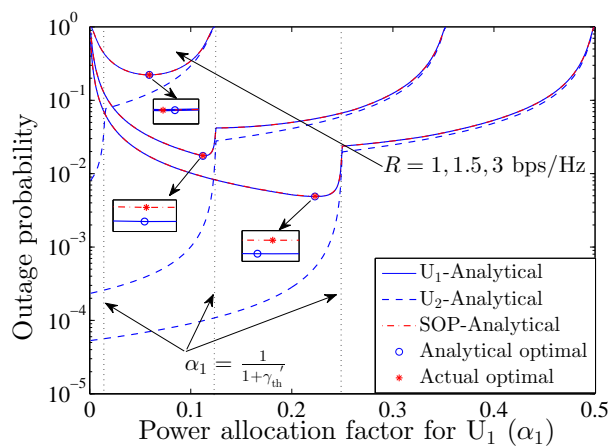

Fig. 3. Outage performance of the ICN protocol for varying $\alpha_{1}\left(\rho_{s}=40 \mathrm{~dB}\right)$.

\section{NUMERICAL RESULTS}

Now numerical investigation is carried out to verify the analytical results and present some non-trivial design insights. Unless otherwise specified, the following parameters are used: $\Omega_{1}=\Omega_{3}=0.1, \Omega_{2}=0.01, \rho_{s}=\rho_{r}$, and $K=10$.

Fig. 2 compares outage performance of the proposed ICN protocol against the $\mathrm{CCN}$ protocol. ${ }^{3}$ A close match between the analytical and simulation results in Fig. 2 verifies the accuracy of our analysis. Fig. 2 also shows that both the ICN and $\mathrm{CCN}$ protocols achieve a full diversity order for each user. Further, we can observe that the proposed ICN protocol is superior to the CCN protocol in terms of each user's OP and the SOP, which is consistent with our analysis in Section III-B.

We define performance gain of the ICN protocol relative to the $\mathrm{CCN}$ protocol as $\mathrm{G}(\%)=100 \times\left(1-\frac{P_{\triangle}^{\mathrm{ICN}}}{P_{\Delta}^{\mathrm{CCN}}}\right)$, where $\Delta \epsilon$ $\{1,2,1 \& 2\}$. In our numerical results with $\alpha_{1}=0.2, R=1$, and $\rho_{s}=30 \mathrm{~dB}$, performance gains of $\mathrm{U}_{1}, \mathrm{U}_{2}$, and the system are $(12.3,17.7,11.9)$ when $\Omega_{2}=0.001,(46.6,68.5,46.8)$ when $\Omega_{2}=0.005$, and $(55.0,78.8,55.2)$ when $\Omega_{2}=0.01$. It is obvious that $\mathrm{U}_{2}$ has the highest performance gain, while the performance gains of $\mathrm{U}_{1}$ and the system are almost the same. Note that this observation is also verified by Fig. 2. All the performance gains shrink as $\Omega_{2}$ decreases, because $\mathrm{S}$ in the ICN protocol tends to transmit information in the CNT mode as the channel from $\mathrm{S}$ to $\mathrm{U}_{2}$ deteriorates.

\footnotetext{
${ }^{3}$ Here we compare our ICN protocol with the $\mathrm{CCN}$ protocol as only statistical CSI is needed in both protocols. If global instantaneous CSI is available, better outage performance can be achieved (e.g., the R-NB protocol with optimal block length allocation in [4]).
}

Fig. 3 investigates the impact of power allocation factor $\alpha_{1}$ on the outage performance of the network. It can be observed that the OP of $\mathrm{U}_{2}$ increases with $\alpha_{1}$, while the OP of $\mathrm{U}_{1}$ first decreases and then increases with $\alpha_{1}$. The reasons are as follows. With a higher $\alpha_{1}, \alpha_{2}$ is lower, and thus, the chance that $\mathrm{U}_{2}$ can successfully decode its information in the DNT mode is lower. Further, in the CNT mode, a lower $\alpha_{2}$ means the chance that $\mathrm{U}_{1}$ correctly decodes $\mathrm{U}_{2}$ 's message is lower, and thus, the chance that $\mathrm{U}_{1}$ can help $\mathrm{U}_{2}$ to achieve $\mathrm{U}_{2}$ 's target rate is lower. Therefore, the $\mathrm{OP}$ of $\mathrm{U}_{2}$ increases with $\alpha_{1}$. The $\mathrm{OP}$ of $\mathrm{U}_{1}$ is affected by two factors as follows. Factor 1: A higher $\alpha_{1}$ means more power for $\mathrm{U}_{1}$ 's signal, which tends to decrease its OP. Factor 2: As aforementioned, a higher $\alpha_{1}$ also means the chance that $\mathrm{U}_{1}$ correctly decodes $\mathrm{U}_{2}$ 's message is lower, or in other words, the chance that $\mathrm{U}_{1}$ performs SIC is lower, which tends to increase $\mathrm{U}_{1}$ 's $\mathrm{OP}$. When $\alpha_{1}$ is low, Factor 1 dominates, and thus, $\mathrm{U}_{1}$ 's OP decreases with $\alpha_{1}$. When $\alpha_{1}$ increases beyond a point, Factor 2 dominates, and thus, $\mathrm{U}_{1}$ 's OP increases with $\alpha_{1}$. From Fig. 3, we can see that the analytical approximation of the optimal $\alpha_{1}$ (which minimizes $P_{1 \text {,asy }}^{\mathrm{ICN}}$ ) is close to the actual optimal value (which is the point of $\alpha_{1}$ that minimizes the SOP). It is worth noticing that when $R=3 \mathrm{bps} / \mathrm{Hz}$, the optimal $\alpha_{1}$ lies in the region $\left[\frac{1}{1+\gamma_{\mathrm{th}}^{\prime}}, \frac{1}{1+\gamma_{\mathrm{th}}}\right)$, which indicates that to minimize SOP, the system should stay in the DNT mode in this case. When $R=1 \mathrm{bps} / \mathrm{Hz}$ and $R=1.5 \mathrm{bps} / \mathrm{Hz}$, the optimal $\alpha_{1}$ is smaller than $\frac{1}{1+\gamma_{\text {th }}^{\prime}}$, and thus, the best system outage performance is achieved by adaptively switching its transmission mode according to the quality of direct link to $\mathrm{U}_{2}$.

\section{CONCLUSION}

We have proposed a cooperative protocol for TUDN networks. We have analytically proved that the proposed ICN protocol outperforms the CCN protocol. Numerical results have validated our analysis and demonstrated valuable insights.

\section{REFERENCES}

[1] L. Dai, B. Wang, Y. Yuan, S. Han, C.-L. I, and Z. Wang, "Nonorthogonal multiple access for 5G: Solutions, challenges, opportunities, and future research trends," IEEE Commun. Mag., vol. 53, no. 9, pp. 7481, Sep. 2015.

[2] New study item proposal: study on non-orthogonal multiple access for NR. 3GPP RP-170829, Mar. 2017.

[3] Z. Ding, M. Peng, and H. V. Poor, "Cooperative non-orthogonal multiple access in 5G systems," IEEE Commun. Lett., vol. 19, no. 8, pp. 1462 1465, Aug. 2015.

[4] Y. Hu, M. C. Gursoy, and A. Schmeink, "Efficient transmission schemes for low-latency networks: NOMA vs. relaying," in Proc. IEEE PIMRC, Oct. 2017, pp. 1-6.

[5] J. N. Laneman, D. N. C. Tse, and G. W. Wornell, "Cooperative diversity in wireless networks: Efficient protocols and outage behavior," IEEE Trans. Inf. Theory, vol. 50, no. 12, pp. 3062-3080, Dec. 2004.

[6] Y. Liu, Z. Ding, M. Elkashlan, and H. V. Poor, "Cooperative nonorthogonal multiple access with simultaneous wireless information and power transfer," IEEE J. Sel. Areas Commun., vol. 34, no. 4, pp. 938 953, Apr. 2016

[7] N. T. Do, D. B. D. Costa, T. Q. Duong, and B. An, "A BNBF user selection scheme for NOMA-based cooperative relaying systems with SWIPT," IEEE Commun. Lett., vol. 21, no. 3, pp. 664-667, Mar. 2017.

[8] M. Abramowitz and I. A. Stegun, Handbook of Mathematical Functions with Formulas, Graphs, and Mathematical Tables. New York, NY, USA: Dover, 1972.

[9] S. Neumark, Solution of Cubic and Quartic Equations. Oxford: Pergamon Press, 1965. 